\title{
Lichen diversity in the managed forests of the Karnieszewice Forest Division and its surroundings (N Poland)
}

\author{
Wiesław Faltynowicz', Agnieszka Kowalewska ${ }^{2}$, Rafal Szymczyk ${ }^{3}$, Martin Kukwa ${ }^{4}$, Edyta Adamska ${ }^{5}$, \\ Pawel Czarnota $^{6}$, Dariusz Kubiak ${ }^{7}$, Katarzyna Pietrzykowska-Urban ${ }^{1}$
}

\author{
${ }^{1}$ Department of Botany, Institute of Environmental Biology, Wrocław University, \\ Kanonia 6/8, 50-328 Wrocław, Poland; e-mail: wieslaw.faltynowicz@uni.wroc.pl \\ ${ }^{2}$ Pomorski Group of Lanscape Parks in Słupsk, Gdańsk Division - Trójmiejski Landscape Park, \\ Polanki 51, 80-308 Gdańsk, Poland \\ ${ }^{3}$ Environmental Survey Laboratory Ekoprojekt, \\ Nowica 24, 14-405 Wilczęta, Poland \\ ${ }^{4}$ Department of Plant Taxonomy and Nature Protection, Gdańsk University, \\ Wita Stwosza 59, 80-308 Gdańsk, Poland \\ ${ }^{5}$ Nicolaus Copernicus University, Faculty of Biology and Environment Protection, \\ Chair of Geobotany and Landscape Planning \\ Lwowska 1, 87-100 Toruń, Poland \\ ${ }^{6}$ Department of Agroecology, Rzeszów University, \\ Ćwiklińskiej 1A, 35-601 Rzeszów, Poland \\ ${ }^{7}$ Department of Mycology, Warmia and Mazury University in Olsztyn, \\ Oczapowskiego 1A, 10-719 Olsztyn, Poland
}

\begin{abstract}
The lichen biota of the Karnieszewice Forest Division (N Poland) is presented. Despite it is predominantly a strongly managed woodland area, 270 lichen species were found there including many rare species for Poland, as well as for European Lowland. Near $20 \%$ of the whole lichen biota are considered to be threatened in the country (categories CR, EN, VU), and 34 species are protected by law in Poland. Agonimia flabelliformis is reported for the second time from Polish lowlands.
\end{abstract}

Key words: biodiversity, lichenized fungi, endangered species, Pomerania, Ascomycota, Basidiomycota, rare species.

\section{Introduction}

Sensitivity of lichens against changes in habitat conditions is known and widely described in literature, beginning with the classical work of Nylander (1866) and thousands books and articles, (e.g. Hawksworth 1971; Herzig \& Urech 1991; Faltynowicz 1995; van Herk 2001; Zalewska 2012; Adamska 2014; Jóźwiak 2014). Also impact of forest management on lichen biota have been mentioned in many publications. In 1934 dr. F. Krawiec wrote: „...Flora on trees in some cases better reflect forest state than vascular plants...in lichen flora distinctly is seen impact of cutting down of forest" (Krawiec 1934), and prof. J. Motyka observed that „List of lichen species is one of the best features of plant communities primarity" (Motyka 1934). Problems of the negative impact of forestry culture on lichens has been widely discussed, e.g. by Czyżewska (1976), Cieśliński (2003), Łubek (2007) and Zalewska (2012), however, despite of the antropogenic changes in forests, in many such areas, particularly in northern Po- 
land, numerous stenotopic or forest relict lichens have been more and more often found (e.g., Cieśliński 2003; Zalewska et al. 2004a, b; Wieczorek 2005; Zalewska 2012; Kubiak et al. 2014). It may be a proof for the improvement of habitat conditions in forests, but it should be further investigated in the future.

The aim of this work was to know a lichen diversity in managed lowland forests and to compare the list of species with results of similar investigations made in other lowland woodlands in Poland including the old and long-term preserved forest areas.

\section{Study area}

The area of Karnieszewice Forest Division has very rich and diversified geomorphology. Ground moraines dominate, but almost all geomorphological forms, typical for the zone of the last glaciation in northern Poland (e.g., frontal moraines, pravalleys, narrow erosion valleys with steep slopes, numerous spring areas) are present here. Landscape is enriched by seashore with mosaices of dunes, sandbars, shore of large lakes and peatbogs.

Such geomorphology gives a great diversity of habitats for plant communities. Coniferous habitats dominate in the forests (nearly 45\%), however other forest associations are also known from this area: Melico-Fagetum, Luzulo pilosae-Fagetum, Stellario-Carpinetum, Ribeso nigri-Alnetum, and smalles areas covered by phytocoenoses of Betuletum pubescentis, Circaeo-Alnetum and Betulo-Quercetum. Non-forest associations important for the occurrence of lichens are mainly Helichryso-Jasionetum and heaths in coniferous complexes.

Tree stands in the Karnieszewice Forest Division posses relatively high average of age (65 years). The dominant tree species is Pinus sylvestris (52\%), and a great share has also Picea abies (9\%). Decidous trees are almost $40 \%$ of forests, with dominating Fagus sylvatica (14\%), Betula spp. (10,5\%), Alnus glutinosa (6\%) and Quercus spp. $(5.5 \%)$. Trees and shrubs growing along roadsides are also very important for lichens, with the most frequent: Acer platanoides, A. pseudoplatanus, Carpinus betulus, Fraxinus excelsior, Corylus avellana, Larix spp., Padus avium, Populus spp., Sorbus aucuparia and Tilia cordata.

More than a half of the forests of Karnieszewice Forest Division (10 $560 \mathrm{ha}$ ) is protected in different ways: Nature 2000 areas, nature reserves, areas of protected landscape, etc. Since 2011 the whole Karnieszewice Forest Division is the part of Forest Promotion Complex „Lasy Środkowopomorskie".

The study area includes also areas not belonging to the Karnieszewice Forest Division - agriculture and rural areas. They are places with rich and completely different lichen biota. Particularly interesting habitats were roadside trees (most often maple, sycamore and lime trees) and trees around old churches.

\section{Materials and methods}

The article is based on observations and collections made during field studies in 2013 (localities nos 1-23; see Kukwa et al. 2013) and 2014 (localities nos 24-53 and 60-69), and on published data (Erichsen 1936, 1940; Tobolewski 1964, 1966, 1979, 1980, 1981; Fałtynowicz 1992; Izydorek 2010 - localities nos 54-59). Specimens are deposited in the herbaria UGDA, GPN, OLS-L, OLTC and WRSL. Lichen substances were analyzed by standard technique of thin-layer chromatography in solvent systems A, B, C and $\mathrm{G}$ (Orange et al. 2001). The nomenclature follows mostly Fałtynowicz and Kukwa (2006), excluding lichens from Teloschistaceae family (Arup et al. 2013) and genera: Alyxoria (Ertz \& Tehler 2011), Caeruleum (Arcadia \& Knudsen 2012), Circinaria (Nordin et al. 2010), Graphis (Neuwirth \& Aptroot 2011), Varicellaria (Schmitt et al. 2012), Violella (Spribille et al. 2011) and Zwackhia (Ertz \& Tehler 2011), and species: Brianaria sylvicola (Ekman \& Svensson 2014), Cladonia floerkeana (Santesson et al. 2004), Lecanora filamentosa (Palice et al. 2011), L. pannonica (Brodo et al. 1994), L. saxicola (Laundon 2010), Lepraria finkii (Lendemer 2011), Melanelixia glabratula (Arup \& Sandler Berlin 2011), Punctelia jeckeri (Kalb 2007), Rinodina oleae (Santesson et al. 2004) and Usnea dasopoga (Arcadia 2013).

Categories of threat follow Cieśliński et al. (2006) and are included in text and Table 2: CR - critically endangered, EN - endangered, VU - vulnerable. Following abbreviations are used: for. - forest district; fs - forest section; Ah - Aesculus hippocastanum, $\mathrm{Al}$ - Alnus glutinosa; Apl - Acer platanoides; Aps - Acer pseudoplatanus; Bet - Betula spp.; Cav - Corylus avellana; $\mathrm{Cb}-$ Carpinus betulus; dw - decaying wood; Fr - Fraxinus excelsior; Fs - Fagus sylvatica; La - Larix spp.; Pa - Picea abies; Pav - Padus avium; Pm - Pseudotsuga menziesii; Ps - Pinus sylvestris; $\mathrm{Pt}$ - Populus tremula; $\mathrm{Ra}$ - Ribes alpinum, $\mathrm{Rr}$ - Rosa rugosa, Q - Quercus spp.; Sal - Salix spp.; Sor Sorbus aucuparia; Tc - Tilia cordata; gl - soil.

\section{List of localities:}

$1-54^{\circ} 13.690^{\prime} \mathrm{N}, 16^{\circ} 20.130^{\prime} \mathrm{E}$, Kacza Island;

$2-54^{\circ} 13.274^{\prime} \mathrm{N}, 16^{\circ} 25.874^{\prime} \mathrm{E}$, for. Sieciemin, fs $421 \mathrm{~b}$, pine forest;

$3-54^{\circ} 12.817^{\prime} \mathrm{N}, 16^{\circ} 26.971^{\prime} \mathrm{E}$, for. Sieciemin, fs $421 \mathrm{c}$, pine forest;

$4-54^{\circ} 14.490^{\prime} \mathrm{N}, 16^{\circ} 29.401^{\prime} \mathrm{E}$, for. Sieciemin, fs $221 \mathrm{f}$, beech forest; 
$5-54^{\circ} 14.333^{\prime} \mathrm{N}, 16^{\circ} 29.587^{\prime} \mathrm{E}$, for. Sieciemin, fs 246 , beech forest;

$6-54^{\circ} 10.002^{\prime} \mathrm{N}, 16^{\circ} 29.444^{\prime} \mathrm{E}$, for. Sieciemin, fs $411 \mathrm{c}$, mixed forest;

$7-54^{\circ} 15.262^{\prime} \mathrm{N}, 16^{\circ} 34.701^{\prime} \mathrm{E}$, for. Lejkowo, trees along road, buildings;

$8-54^{\circ} 15.564^{\prime} \mathrm{N}, 16^{\circ} 34.347^{\prime} \mathrm{E}$, for. Lejkowo, fs $115 \mathrm{a}$ and $111 \mathrm{a}$, water-headwith marshy meadow, beech forest;

$9-54^{\circ} 12.345^{\prime} \mathrm{N}, 16^{\circ} 34.070^{\prime} \mathrm{E}$, for. Lejkowo, fs 152 , mixed forest;

$10-54^{\circ} 11.333^{\prime} \mathrm{N}, 16^{\circ} 27.156^{\prime} \mathrm{E}$, for. Szczeglino, fs $563 \mathrm{f}$, beech forest and stones on cemetary;

$11-54^{\circ} 11.561^{\prime} \mathrm{N}, 16^{\circ} 30.862^{\prime} \mathrm{E}$, trees along road between villages Ratajki and Sowno;

$12-54^{\circ} 12.585^{\prime} \mathrm{N}, 16^{\circ} 27.774$ 'E, Ratajki village, church and trees;

$13-54^{\circ} 14.626^{\prime} \mathrm{N}, 16^{\circ} 22.221^{\prime} \mathrm{E}$, for. Sianów, fs $192 \mathrm{a}$;

$14-54^{\circ} 13.354^{\prime} \mathrm{N}, 16^{\circ} 20.864^{\prime} \mathrm{E}$, for. Sianów, fs $240 \mathrm{c}$;

$15-54^{\circ} 17.772^{\prime} \mathrm{N}, 16^{\circ} 16.589^{\prime} \mathrm{E}$, Iwięcino, church;

$16-54^{\circ} 18.252^{\prime} \mathrm{N}, 16^{\circ} 13.488^{\prime} \mathrm{E}$, for. Iwięcino, “'Łazy” nature reserve, fs $673 \mathrm{f}$, peatbog with pine forest;

$17-54^{\circ} 18.333^{\prime} \mathrm{N}, 16^{\circ} 13.688^{\prime} \mathrm{E}$, for. Iwięcino, fs $673 \mathrm{~b}$, beech forest;

$18-54^{\circ} 13.713^{\prime} \mathrm{N}, 16^{\circ} 09.068^{\prime} \mathrm{E}$, for. Chełmoniewo, fs $383 \mathrm{~b}$, mixed forest;

$19-54^{\circ} 15.710^{\prime} \mathrm{N}, 16^{\circ} 25.919^{\prime} \mathrm{E}$, for. Niemica, fs $99 \mathrm{c}$, peatbog and pine forest;

$20-54^{\circ} 11.541^{\prime} \mathrm{N}, 16^{\circ} 16.377^{\prime} \mathrm{E}$, for. Koszalin, fs $332 \mathrm{f}$, $343 \mathrm{~b}, \mathrm{c}, \mathrm{d}, 333 \mathrm{~h}, \mathrm{~d}$; beech forest;

$21-54^{\circ} 16.809^{\circ} \mathrm{N}, 16^{\circ} 22.043^{\circ} \mathrm{E}$, for. Kamionka, fs $19 \mathrm{~b}$;

$22-54^{\circ} 16.215^{\circ} \mathrm{N}, 16^{\circ} 19.693$,E, Arboretum;

$-54^{\circ} 14.474^{\prime} \mathrm{N}, 16^{\circ} 20.837^{\prime} \mathrm{E}$, Trawica, builgings of the Karnieszewice upper-forestry;

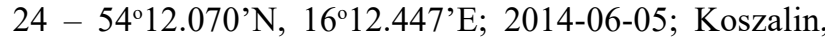
beech forest;

$25-54^{\circ} 12.272^{\prime} \mathrm{N}, 16^{\circ} 13.313^{\prime} \mathrm{E}$; beech forest and walls;

$26-54^{\circ} 11.420^{\prime} \mathrm{N}, 16^{\circ} 17.465^{\prime} \mathrm{E}$; for. Chełmoniewo, fs $401 \mathrm{~A} / \mathrm{C}$, mixed forest;

$27-54^{\circ} 11.408^{\prime} \mathrm{N}, 16^{\circ} 17.333^{\prime} \mathrm{E}$; for. Chełmoniewo, fs $392 \mathrm{~g}$, mixed forest;

$28-54^{\circ} 11.406^{\prime} \mathrm{N}, 16^{\circ} 17.540^{\prime} \mathrm{E}$; for. Chełmoniewo, mixed forest;

$29-54^{\circ} 11.415^{\prime} \mathrm{N}, 16^{\circ} 17.506^{\prime} \mathrm{E}$; for. Chełmoniewo, mixed forest;

$30-54^{\circ} 12.287^{\prime} \mathrm{N}, 16^{\circ} 16.226^{\prime} \mathrm{E}$; beech forest;

$31-54^{\circ} 12.270^{\prime} \mathrm{N}, 16^{\circ} 15.488^{\prime} \mathrm{E}$; peatbog, trees and stones;

$32-54^{\circ} 12.542^{\prime} \mathrm{N}, 16^{\circ} 16.251^{\prime} \mathrm{E}$; trees along road;

$33-5^{\circ} 12.159^{\prime} \mathrm{N}, 16^{\circ} 30.160^{\prime} \mathrm{E}$; mixed forest;

$34-54^{\circ} 12.145^{\prime} \mathrm{N}, 16^{\circ} 30.039^{\prime} \mathrm{E}$; Dolina Białki, mixed forest and beech forest;

$35-54^{\circ} 12.180^{\prime} \mathrm{N}, 16^{\circ} 29.508^{\prime} \mathrm{N}$; Dolina Białki, mixed forest, stones in the stream;

$36-54^{\circ} 12.344^{\prime} \mathrm{N}, 16^{\circ} 29.473^{\prime} \mathrm{E}$; oak forest;

$37-54^{\circ} 13.066^{\prime} \mathrm{N}, 16^{\circ} 29.467^{\prime} \mathrm{E}$; mixed forest and erratic; $38-54^{\circ} 13.030^{\prime} \mathrm{N}, 16^{\circ} 29.222^{\prime} \mathrm{E}$;mixed forest;

$39-54^{\circ} 13.161^{\prime} \mathrm{N}, 16^{\circ} 29.462^{\prime} \mathrm{E}$; pine forest;

$40-54^{\circ} 13.240^{\prime} \mathrm{N}, 16^{\circ} 30.490^{\prime} \mathrm{E}$; trees along road;

$41-54^{\circ} 14.313^{\prime} \mathrm{N}, 16^{\circ} 32.553^{\prime} \mathrm{E}$; trees along road and stones;

$42-54^{\circ} 12.038^{\prime} \mathrm{N}, 16^{\circ} 27.447^{\prime} \mathrm{E}$; Kościernica village, trees along road and stones;

43 - 54 $11.094^{\prime} \mathrm{N}, 16^{\circ} 20.428^{\prime} \mathrm{E}$; peatbog;

$44-54^{\circ} 11.059^{\prime} \mathrm{N}, 16^{\circ} 20.361^{\prime} \mathrm{E}$; peatbog;

$45-54^{\circ} 10.266^{\prime} \mathrm{N}, 16^{\circ} 23.385^{\prime} \mathrm{E}$; Maszkowo village, trees along road;

$46-54^{\circ} 08.372^{\prime} \mathrm{N}, 16^{\circ} 29.434^{\prime} \mathrm{E}$; pine forest;

$47-54^{\circ} 08.578^{\prime} \mathrm{N}, 16^{\circ} 30.019^{\prime} \mathrm{E}$; pine forest and wall;

$48-54^{\circ} 11.132^{\prime} \mathrm{N}, 16^{\circ} 31.056^{\prime} \mathrm{E}$; trees along road;

$49-54^{\circ} 11.515^{\prime} \mathrm{N}, 16^{\circ} 34.134^{\prime} \mathrm{E}$; trees along road;

$50-54^{\circ} 11.024^{\prime} \mathrm{N}, 16^{\circ} 33.394^{\prime} \mathrm{E}$; Sierakowo Słowieńskie village, church, walls, stones;

$51-54^{\circ} 13.302^{\prime} \mathrm{N}, 16^{\circ} 31.246^{\prime} \mathrm{E}$; trees, wall, stones;

$52-54^{\circ} 12.112^{\prime} \mathrm{N}, 16^{\circ} 30.157^{\prime} \mathrm{E}$; buildings, trees,

$53-54^{\circ} 18.585^{\prime} \mathrm{N}, 16^{\circ} 12.315^{\prime} \mathrm{E}$; Spit of Kopań Lake;

$54-54^{\circ} 15.331^{\prime} \mathrm{N}, 1^{\circ} 02.317^{\prime} \mathrm{E}$; Mielno town;

$55-54^{\circ} 15.175^{\prime} \mathrm{N}, 16^{\circ} 00.452^{\prime} \mathrm{E}$; between Mielno and Chłopy;

$56-54^{\circ} 18.579^{\prime} \mathrm{N}, 16^{\circ} 12.351^{\prime} \mathrm{E}$; Łazy village, between Jamno Lake and Bukowo Lake;

$57-54^{\circ} 16.384^{\prime} \mathrm{N}, 16^{\circ} 06.559^{`} \mathrm{E}$; Unieście village, on concrete and Quercus;

$58-54^{\circ} 17.044^{\prime} \mathrm{N}, 16^{\circ} 16.153^{\prime} \mathrm{E}$; Iwięcino village, ca. $1 \mathrm{~km}$ on $\mathrm{W}$, Acer pseudoplatanus trees along road;

$59-54^{\circ} 14.562^{\prime} \mathrm{N}, 15^{\circ} 57.121^{\prime} \mathrm{E}$; Sarbinowo village, $\lg$. Flössner 1935 (Erichsen 1940).

$60-54^{\circ} 16.243^{\prime} \mathrm{N}, 16^{\circ} 32.258^{\prime} \mathrm{E}$; between Bartolino and Sulechowo villages, Acer platanoides trees along road;

$61-54^{\circ} 16.157^{\prime} \mathrm{N}, 16^{\circ} 33.031^{\prime} \mathrm{E}$; Sulechówko village, near church; stones and different species of trees;

$62-54^{\circ} 15.304^{\prime} \mathrm{N}, 16^{\circ} 34.269^{\prime} \mathrm{E}$; between Kukułczyn and Lejkowo villages, wood and different species of trees;

63 - 54 $16.446^{\prime} \mathrm{N}, 16^{\circ} 18,407^{\prime} \mathrm{E}$; Wierciszewo village, limes along road and wall;

$64-54^{\circ} 22.284^{\prime} \mathrm{N}, 16^{\circ} 18,236^{\prime} \mathrm{E}$; Spit of Bukowo Lake, Dąbki village, ca. $1 \mathrm{~km}$ on $\mathrm{W}$; different species of trees; $65-54^{\circ} 21.495^{\prime} \mathrm{N}, 16^{\circ} 17.083^{\prime} \mathrm{E}$; Spit of Bukowo Lake, Dąbki village, ca. $2 \mathrm{~km}$ on $\mathrm{W}$; different species of trees; $66-54^{\circ} 16.363^{\prime} \mathrm{N}, 16^{\circ} 13.222^{\prime} \mathrm{E}$; between Kleszcze and Osieki villages; maples along road and concrete;

$67-54^{\circ} 17.010^{\prime} \mathrm{N}, 16^{\circ} 12.390^{\prime} \mathrm{E}$; Osieki village, church, stones, wall, ashes and beeches;

$68-54^{\circ} 18.019^{\prime} \mathrm{N}, 16^{\circ} 10.428^{\prime} \mathrm{E}$; Spit of Jamno Lake, Łazy village, ca. $1,5 \mathrm{~km}$ on $\mathrm{W}$. 


\section{Results and discussion}

Lichenological data from the Karnieszewice Forest Division and adjacent areas are relatively insufficient. The oldest, single information is comprised in publications of Erichsen (1936, 1940), Tobolewski (1964, 1966, 1979, 1980, 1981) and Fałtynowicz (1992). Several species from south-east part of Karnieszewice Forest Division have been reported by Izydorek (2010), and a brief information about Koszalin City lichens can be found in the conference abstract by Bezmian (1998). The most complete data have been published recently by Kukwa et al. (2013), however those records represented only preliminary results.

Altogether 270 lichen species were found in the explored area. Such a large number of species is apparently a result related to the great diversity of substrates and habitats in this area. This number of species is relatively high also for the Polish Lowland (Table 1). There is no doubts it is also a result of the relatively good condition of forest associations, which despite intensive forest cultivation (from $19^{\text {th }}$ century), preserved their values and specific phytoclimate.

In comparision to other protected areas in northern Poland with similar acreage and partly also habitats, the Karnieszewice Forest Division is one of the richest; also numerous red-listed species (Table 2) and taxa protected by law (Table 3) occur there. Epiphytic lichens dominate in the lichen biota (165 species), but the group of wood-inhabiting species is also rich ( 75 species). Epilithic lichens are also numerous (69 species), however, on the natural substrate (boulders and stones) only 47 species have been found. On antropogenic substrate (concrete, bricks, tiles, eternite) 38 further species have been recorded. The biota of terricolous lichens includes 45 species (most of them belong to Cladonia genus); this group of lichens does not have suitable habitats in the study area. In addition seven species were also found on metal substrate.

The importance of the Karnieszewice Forest Division for the lichen diversity protection can be also proved by the very long list of lichen taxa with the highest categories of threat in Poland. 53 such species have been found $(19,6 \%$ of the whole local biota) (Table 2), five of which with CR category and 21 species with EN category. Among them there are taxa particularly rare in the country, such as Cladonia parasitica and Ramalina baltica, but some of them occur very frequently in northern Poland thus in studied area their category of threat is surely lower. It is applicable among the others for Cladonia caespitica, Flavoparmelia caperata, Pleurosticta acetabulum, Ramalina fastigiata, $R$. fraxinea and Usnea florida. Agonimia flabelliformis is reported for the second time from Polish lowland (Kowalewska unpubl. data).

In the Karnieszewice Forest Division 34 species protected by law were found (Table 3).
Table 1. The numer of lichen species known from selected protected areas in N Poland with reference to the Karnieszewice Forest Division

\begin{tabular}{|l|c|}
\hline Name of protected area & $\begin{array}{c}\text { Number } \\
\text { of species }\end{array}$ \\
\hline Białowieski National Park (Cieśliński 2003) & ca. 360 \\
\hline Wigierski National Park (Fałtynowicz 2014) & 303 \\
\hline Wdzydzki Landscape Park (Kukwa et al. 2012) & 276 \\
\hline Karnieszewice Forest Division & 270 \\
\hline $\begin{array}{l}\text { Słowiński National Park (Fałtynowicz 2015, } \\
\text { unpbl.) }\end{array}$ & 270 \\
\hline Woliński National Park (Wieczorek 2014) & 266 \\
\hline $\begin{array}{l}\text { Drawieński National Park (Schiefelbein et al. } \\
\text { 2012) }\end{array}$ & 262 \\
\hline Suwalski Landscape Park (Zalewska et al. 2004a) & 231 \\
\hline $\begin{array}{l}\text { Landscape Park of Puszcza Romincka (Zalewska } \\
\text { et al. 2004b) }\end{array}$ & 222 \\
\hline Szczeciński Landscape Park (Wieczorek 2005) & 207 \\
\hline
\end{tabular}

\section{List of species}

In the list numbers of sites and substrate type were noted for each species; + - non-lichenized fungus

Absconditella lignicola Vězda \& Pišút - 3, 34, 35, 38, 39; Al, Ps, dw;

A. sphagnorum Vězda \& Poelt - 43, 44; on Sphagnum spp. Acarospora fuscata (Nyl.) Th. Fr. - 12, 15, 31, 50, 51; stones, wall;

A. moenium (Vain.) Räs. - 47; wall;

Acrocordia gemmata (Ach.) A. Massal. - 3; Pt;

Agonimia flabelliformis Halda, Czarnota \& Guzow-Krzemińska - 35; Al;

Alyxoria varia (Pers.) Ertz \& Tehler - 23; Apl; (Izydorek 2010);

Amandinea punctata (Hoffm.) Coppins \& Scheid. - 9, 11, 40-42, 45, 51, 52, 56, 58, 60, 61, 66; Apl, Aps, Bet, Fr, Pa, Q; (Izydorek 2010);

Anaptychia ciliaris (L.) Körb. - 61; Apl;

Anisomeridium polypori (M.B. Ellis \& Everh.) M.E. Barr - 3, 32, 48; Apl, Fr, Pt;

Arthonia didyma Körb. - 33; Cb;

A. mediella Nyl. - 20, 29; Q; (Izydorek 2010);

A. radiata (Pers.) Ach. - 9, 32, 33, 35, 37, 39, 54, 64; Apl, Bet, Cav, Cb, Fr, Fs, Ps, Q, Sal, Sor;

A. spadicea Leigh. - 3, 8, 10, 26, 27, 30-33, 36-38, 54, 64, 65; Al, Cav, Cb, Fr, Pav, Ps, Q, Tc; 
Table 2. Species from Polish Red List of Lichens (Cieśliński et al. 2006) found in the Karnieszewice Forest Division

\begin{tabular}{|c|c|c|c|}
\hline $\begin{array}{l}\text { Categories } \\
\text { of threats }\end{array}$ & Name of species & $\begin{array}{c}\text { Number of species/ } \\
\text { Total number of } \\
\text { localities }\end{array}$ & $\begin{array}{l}\% \text { of } \\
\text { lichen } \\
\text { biota }\end{array}$ \\
\hline CR & $\begin{array}{l}\text { Bryoria cf. capillaris, Chrysothrix candelaris, Melanohalea olivacea, Pertusaria } \\
\text { hymenea, Pyrrhospora quernea }\end{array}$ & $5 / 9$ & 1.8 \\
\hline EN & $\begin{array}{l}\text { Anaptychia ciliaris, Arthonia didyma, Calicium adspersum, Caloplaca chlorina, } \\
\text { Cetraria sepincola, Chaenotheca brunneola, Ch. stemonea, Cladonia caespiticia, C. } \\
\text { parasitica, Fellhanera bouteilei, Flavoparmelia caperata, Lecanora intumescens, } \\
\text { Opegrapha atra, Pertusaria flavida, Physconia distorta, Ph. perisidiosa, Pleurosticta } \\
\text { acetabulum, Ramalina baltica, R. fastigiata, R. fraxinea, Usnea florida }\end{array}$ & $21 / 90$ & 7.8 \\
\hline VU & $\begin{array}{l}\text { Acrocordia gemmata, Arthonia mediella, Bacidia rubella, Biatora efflorescens, } \\
\text { Bryoria fuscescens, Calicium glaucellum, C. salicinum, C. viride, Cetraria } \\
\text { chlorophylla, C. islandica, Cliostomum griffithii, Melanohalea elegantula, } \\
\text { Ochrolechia androgyna, Opegrapha rufescens, O. vulgata, Parmelia submontana, } \\
\text { Parmelina tiliacea, Peltigera canina, P. praetextata, Pertusaria pertusa, Punctelia } \\
\text { subrudecta, Pyrenula nitida, Ramalina farinacea, Usnea dasopoga, U. hirta, } \\
\text { Varicellaria hemisphaerica, Zwackhia viridis }\end{array}$ & $27 / 136$ & 10 \\
\hline \multicolumn{2}{|l|}{ Total } & $53 / 235$ & 19.6 \\
\hline
\end{tabular}

Table 3. Lichen species protected by Polish law (Rozporządzenie Ministra Środowiska 2014) in the Karnieszewice Forest Division

\begin{tabular}{|c|c|c|c|}
\hline $\begin{array}{l}\text { Protection } \\
\text { forms }\end{array}$ & Name of species & $\begin{array}{l}\text { Number of species/ } \\
\text { Total number of } \\
\text { localities }\end{array}$ & $\begin{array}{l}\% \text { of } \\
\text { lichen } \\
\text { biota }\end{array}$ \\
\hline $\begin{array}{l}\text { Total } \\
\text { protection }\end{array}$ & $\begin{array}{l}\text { Anaptychia ciliaris, Bryoria } \text { cf. capillaris, Cetraria sepincola, Chrysothrix candelaris, } \\
\text { Melanohalea elegantula, M. olivacea, Parmelia submontana, Parmelina tiliacea, } \\
\text { Peltigera hymenina, Punctelia jeckeri, P. subrudecta, Ramalina baltica, R. fastigiata, } \\
\text { R, fraxinea, Usnea florida, Xanthoparmelia delisei }\end{array}$ & $15 / 70$ & 5.7 \\
\hline $\begin{array}{l}\text { Partly } \\
\text { protected }\end{array}$ & $\begin{array}{l}\text { Bryoria fuscescens, Cetraria islandica, Cladonia arbuscula, C. ciliata, C. portentosa, } \\
\text { C. rangiferina, Flavoparmelia caperata, Hypogymnia tubulosa, Imshaugia aleurites, } \\
\text { Melanelixia subaurifera, Peltigera canina, P. membranacea, P. polydactylon, } \\
\text { Pleurosticta acetabulum, Ramalina farinacea, Usnea dasopoga, U. hirta, Vulpicida } \\
\text { pinastri }\end{array}$ & $18 / 133$ & 7.0 \\
\hline \multicolumn{2}{|l|}{ Total } & $33 / 203$ & 12.7 \\
\hline
\end{tabular}

A. vinosa Leight. - 29, 36-38; Q;

Arthothelium ruanum (A. Massal.) Körb. - 8, 35, 54; Cav, Pav;

Aspicilia cinerea (L.) Körb. - 15, 50; stones;

Athallia holocarpa (Hoffm.) Arup, Frödén \& Søchting 12, 15, 51, 52, 55, 67, 68; walls, stone;

A. pyracea (Ach.) Arup, Frödén \& Søchting - 1; Pt;

Bacidia rubella (Hoffm.) A. Massal. - (Izydorek 2010);

Bacidina sulphurella (Samp.) M. Hauck \& V. Wirth - 24, 26-28, 32-35; Cav, Cb, Fs, Q;

Baeomyces rufus (Huds.) Rebent. - 31, 50; gl, stones; (Izydorek 2010);

Biatora efflorescens (Hedl.) Erichsen - 5, 9, 18, 24, 26, 30, 37; Fr, Fs, Q;
B. globulosa (Flörke) Fr. - 22, 36, 38, 40, 55; Apl, Aps, Fr;

Bilimbia sabuletorum (Schreb.) Arnold - 54; on bryophytes over old wall;

Brianaria sylvicola (Flot. ex Körb.) S. Ekman \& M. Svensson - (Izydorek 2010);

Bryoria cf. capillaris (Ach.) Brodo \& D. Hawksw. - 36; Q; B. fuscescens (Gyelnik) Brodo \& D. Hawksw. - 6, 36, 37, 39, 40, 52, 54, 55; Bet, La, Q, dw;

Buellia alboatra s.1. - 15, 50, 51, 67; stone, walls;

B. griseovirens (Turner \& Borrer ex Sm.) Almb. - 3, 4, 9, $16,18,24,26,27,29,30,33-35,38,42,48,51,52,54$, 67; Al, Bet, Cav, Cb, Fr, Fs, Pt, Q, Sor, dw;

Calicium adspersum Pers. - 28, 37, 38; Q;

C. glaucellum Ach. - 39; dw; 
C. parvum Tibell - 39, 43, 47; Ps;

C. pinastri Tibell - 43, 53; Ps;

C. salicinum Pers. - 20, 29, 35, 36, 38, 40, 53; Q, dw; (Izydorek 2010);

C. viride Pers. - 18, 29, 31, 35, 36, 38; Fr, Q;

Calogaya decipiens (Arnold) Arup, Frödén \& Søchting 3, 15, 51, 55, 63; walls; (Izydorek 2010);

C. pusilla (A. Massal.) Arup, Frödén \& Søchting - 15, 63, 67, 69; walls;

Caloplaca cerina (Ehrh. ex Hedwig) Th. Fr. - 36; dw;

C. chlorina (Flot.) H. Olivier - 15; Fr;

C. obscurella (J. Lahm ex Koerb.) Th. Fr. - 11, 41, 45; Apl, Aps;

C. saxicola (Hoffm.) Nordin - 51, 55, 67; walls;

Candelaria concolor (Dickson) B. Stein - 9, 45, 52; Apl, Aps, Fr, dw;

C. pacifica Westberg - 48, 61, 63; Fr, Tc;

Candelariella aurella (Hoffm.) A. Zahlbr. - 3, 11, 15, 25, 51, 52, 55, 61, 66, 69; Aps, walls; (Izydorek 2010);

C. efflorescens R.C. Harris \& W.R. Buck - 9, 24, 26, 36, 61; Apl, Cav, Fr, Pt;

C. vitellina (Hoffm.) Müll. Arg. - 12, 15, 48, 51; Fr, stones; (Izydorek 2010);

C. xanthostigma (Ach.) Lettau - 11; 15, 40-42, 48, 56; Aps, Apl, Fr, Q; (Izydorek 2010);

Catillaria nigroclavata (Nyl.) Schuler - 24, 26, 36; Pt, Fs, dw;

Cetraria aculeata Ach. - 14; gl; (Izydorek 2010);

C. chlorophylla (Willd.) Vain. - 6, 7, 11, 22, 40, 42, 52, 54, 55, 62; Aps, Bet, La, Pa, Q, metal, dw;

C. islandica (L.) Ach. - 2; gl; (Izydorek 2010);

C. sepincola (Ehrh.) Ach. - 31, 52; Bet; (Izydorek 2010);

Chaenotheca brunneola (Ach.) Müll. Arg. - 29; Q;

Ch. chrysocephala (Ach.) Th. Fr. - 3, 17, 18, 20, 22, 28, 31, 33, 35-38; Al, Bet, Fr, Q, dw;

Ch. ferruginea (Turner ex Sm.) Mig. - 1, 10, 16, 18-20, 22, 26, 28-31, 33-40, 43, 44, 46, 47, 52-55, 61, 64, 67; Al, Aps, Bet, Fr, La, Ps, Q, dw; (Izydorek 2010);

Ch. stemonea (Ach.) Müll. Arg. - 26, 35; dw, Al;

Ch. trichialis (Ach.) Th. Fr. - 36; Bet; (Izydorek 2010);

Chrysothrix candelaris (L.) J.R. Laundon - 17, 28, 29, 37, 38, 61; Fr, Q; (Izydorek 2010);

Circinaria caesiocinerea (Nyl. ex Malbr.) A. Nordin, S. Savić \& Tibell - 51; stones;

C. calcarea (L.) A. Nordin, S. Savić \& Tibell - 52; wall;

C. contorta (Hoffm.) A. Nordin, S. Savić \& Tibell - 3, 25, 51, 52; walls;

Cladonia arbuscula s.1. - 2, 14, 19, 39, 44, 55, 67; gl; (Izydorek 2010);

C. caespiticia (Pers.) Flk. - 29; gl; (Izydorek 2010);

C. cenotea (Ach.) Schaer. - 2, 3, 31, 35, 37, 43; Bet, Q, dw; (Izydorek 2010);

C. cervicornis (Ach.) Flot. ssp. verticillata (Hoffm.) Ahti - 14, 37, 67; gl;
C. chlorophaea (Flörke ex Sommerf.) Spreng. s.str. - 14, 54, 55; Bet, gl; (Izydorek 2010);

C. ciliata Stirt. - 2, 14, 39, 44, 55; gl; between Sphagnum on peatbog;

C. coniocraea (Flörke) Vain. - 1-3, 8-10, 13, 16, 18-22, 24, 25, 27-31, 33-38, 40, 42-44, 46, 47, 52-55, 62, 64, 65, 67; Al, Bet, Fr, Fs, Pa, Ps,Q, gl, dw; (Izydorek 2010);

C. cornuta (L.) Hoffm. - 3, 14, 55; Bet, gl; (Izydorek 2010);

C. deformis (L.) Hoffm. - 31, 46, 47; gl, dw; (Izydorek 2010);

C. digitata (L.) Hoffm. - 1-3, 13, 16, 19, 21, 22, 26, 27, 29, 31, 34-37, 39, 42, 46, 47, 52, 54, 55, 64; Al, Bet, Fs, La, Ps, Q, dw, gl; (Izydorek 2010);

C. fimbriata (L.) Fr. - 3, 16, 19, 27, 29, 31, 32, 40, 45, 52, 54, 55, 62, 67; Apl, Aps, Bet, Ps, dw, gl; (Izydorek 2010);

C. floerkeana (Fr.) Flörke - 3, 14, 27, 46; Bet, gl, dw; (Izydorek 2010);

C. foliacea (Huds.) Willd. - 55; gl;

C. furcata (Huds.) Schrad. - 2, 14, 31, 51-53, 55, 67; gl, dw; (Izydorek 2010);

C. glauca Flörke - 2, 14, 19, 55; gl; (Izydorek 2010);

C. gracilis (L.) Willd. - 14, 39, 55; gl; (Izydorek 2010);

C. grayi G. Merr. ex Sandst. - 2, 3, 26, 31; gl, dw;

C. macilenta Hoffm. - 2, 3, 14, 19, 26, 35, 36, 40, 46, 55; Bet, gl, dw; (Izydorek 2010);

C. novochlorophaea (Sipman) Brodo \& Ahti - 19; gl;

C. parasitica (Hoffm.) Hoffm. - 34, dw;

C. phyllophora Hoffm. - 14, 55; gl; (Izydorek 2010);

C. pleurota (Flörke) Schaer. - 9; Fr;

C. portentosa (Dufour) Coem. - 19, 55, 67; gl; (Izydorek 2010);

C. pyxidata (L.) Hoffm. - 46; gl; (Izydorek 2010);

C. ramulosa (With.) J.R. Laundon - 32; Fr; (Izydorek 2010);

C. rangiferina (L.) Web. - 55; gl; (Izydorek 2010);

C. scabriuscula (Delise) Leight. - 53, 55, 67; gl;

C. squamosa Hoffm. - 26; humus, dw;

C. subulata (L.) Weber ex F.H. Wigg. - 23, 55; gl; (Izydorek 2010);

C. uncialis (L.) Weber ex F.H. Wigg. - 2, 39; gl; (Izydorek 2010);

Cliostomum griffithii (Sm.) Coppins - 18, 53, 64, 65, 67; Apl, Fr, Q;

Coenogonium pineti (Schrad. ex Ach.) Lücking \& Lumbsch - 3, 18, 21,26, 31, 33, 35, 36, 42, 64; Al, Bet, Fs, $\mathrm{Pa}, \mathrm{Ps}$, dw;

Evernia prunastri (L.) Ach. - 2, 3, 7, 9-11, 13, 15, 18-20, 22, 31, 32, 34-40, 42, 49, 51-55, 58, 60, 61, 62, 64-67; Al, Apl, Aps, Bet, Cav, Cr, Fr, Fs, La, Pa, Pm, Po, Ps, Q, Sor, Tc, U, dw, stones, metal; (Izydorek 2010); 
Fellhanera bouteillei (Desm.) Vězda - 37-39, 46; twigs of $\mathrm{La}$ and $\mathrm{Pa}$;

F. subtilis (Vězda) Diederich \& Sérus.- 3, 6, 29, 37, 38, 45; twigs of Vaccinium myrtillus and $\mathrm{Pa}$;

Fellhaneropsis myrtillicola (Erichsen) Hafellner - 29, 37, 38, 45; twigs of $\mathrm{Pa}$;

Flavoparmelia caperata (L.) Hale - 15, 18, 67; Fr, Fs;

Flavoplaca citrina (Hoffm.) Arup, Frödén \& Søchting 15, 52, 57, 61, 67, 69; walls, eternit; (Izydorek 2010);

F. flavocitrina (Nyl.) Arup, Frödén \& Søchting - 15, 51, 52; stone, walls;

Fuscidea arboricola Coppins \& Tønsberg - 3, 8, 35; Bet, $\mathrm{Al}$;

F. pusilla Tønsberg - 2, 3, 31, 33, 35, 36, 38, 39, 43, 44, 46, 53, 64; Bet, Cav, Cb, Pa, Ps;

Graphis betulina (Pers.) Ach. - 4, 8 (cf); Fs;

G. pulverulenta (Pers.) Ach. - 4, 5, 8-10, 17, 21, 26-28, 30-35, 38, 40, 44; Cb, Al, Cav, Fs, Q, Aps, Sor;

G. scripta (L.) Ach. - 28, 30, 32-35, 54; Apl, Cav, Fr, Fs, Pav;

Halecania viridescens Coppins \& P. James - 33; Pav;

Hypocenomyce caradocensis (Leight. ex Nyl.) P. James \& Gotth. Schneid. - 22, 53; Pm, Q;

H. scalaris (Ach.) M. Choisy - 2, 3, 7, 13, 16, 19, 20, 22, 25, 26, 29-31, 35-37, 39, 40, 42-44, 46, 47, 52-55, 62, 64, 66; Al, Apl, Bet, Cav, La, Pa, Ps, Q, Tc; dw, stone; (Izydorek 2010);

Hypogymnia physodes (L.) Nyl. - 3, 9, 10, 13, 14, 16, 18-22, 24, 25, 27, 29, 31, 32, 34-40, 42, 43, 44, 46, 47, 51-55, 58, 61, 62, 64, 67, 68; Al, Aps, Bet, Cav, Fr, Fs, La, Pt, Ps, Pa, Q, Tc; dw, stones, sand; (Izydorek 2010);

H. tubulosa (Schaer.) Havaas - 3, 6, 9, 13, 14, 19, 20, 22, 25, 36, 37-40, 42, 46, 52, 53, 61, 62, 68; Bet, Cav, Fr, Fs, La, Pa, Po, Ps, Q, Sal, dw, stone; (Izydorek 2010);

Imshaugia aleurites (Ach.) S.L.F. Meyer - 2, 3, 19, 31, 36, 39, 40, 43, 44, 47, 53, 55, 62; Bet, Ps, Pm, dw;

Lecania cyrtella (Ach.) Th. - 36, 40; Aps, dw;

L. hyalina (Fr.) R. Sant. - (Izydorek 2010);

L. naegelii (Hepp) Diederich \& P. Boom - 9, 32, 36, 41, 49, 54, 56; Apl, Aps, Fr, Fs, Pt, U; (Izydorek 2010);

Lecanora albella (Pers.) Ach. - (Izydorek 2010);

L. albescens (Hoffm.) Flörke - 3, 12, 15, 25, 51, 52, 55, 67; walls; (Izydorek 2010);

L. allophana Nyl. - 41, 46, 48, 53; Apl, Fr, Cr, Ps;

L. argentata (Ach.) Malme - 8, 9, 18, 29, 33, 35, 40, 45, 48, 54-56, 58; Al, Apl, Aps, Cb, Fr, Fs, Q, dw; (Izydorek 2010);

L. carpinea (L.) Vain. - 1, 9, 33, 35, 36, 41, 49, 53-56, 64; Apl, Cb, Cr, Fr, Pt, Q, U, dw; (Izydorek 2010);

L. chlarotera Nyl. - 2, 9, 11, 15, 22, 32, 33, 36, 42, 45, 48, 49, 52-56, 64; Apl, Aps, Bet, Cr, Fr, Pt, Q, U, dw; (Izydorek 2010);

L. compallens van Herk \& Aptroot - 11, 35, 36; Aps, Cb;
L. conizaeoides Nyl. ex Cromb. - 2, 3, 16, 19, 20, 22, 25, 27, 29, 31, 35, 40, 43, 46, 47, 52-55, 58, 62, 64; Al, Aps, Bet, Fr, La, Pa, Ps, Q, Sor, dw; (Izydorek 2010);

L. dispersa (Pers.) Sommerf. - 15, 51, 52, 55, 57, 61, 63, 66-69; walls; (Izydorek 2010);

L. expallens Ach. - 9, 11, 15, 16, 18, 20, 22, 29-32, 37, 38, 45, 51-55; Apl, Aps, Al, Bet, Fr, Fs, Pa, Q; (Izydorek 2010);

L. filamentosa (Stirt.) Elix \& Palice - 2, 3, 13, 14, 18, 19, 24, 25, 27, 31, 38, 40, 43, 44, 47; Bet, Fr, Ps, dw;

L. glabrata (Ach.) Malme - 22; Fr;

L. hagenii (Ach.) Ach. - 11, 15, 51; Aps, walls;

L. intumescens (Rebent.) Rabenh. - 5, 35, 54; Cb, Fs;

L. pannonica Szat. - 15; wall;

L. persimilis (Th. Fr.) Nyl. - 45, 52; Apl, Pa;

L. polytropa (Ehrh.) Rabenh. - 12, 41, 50, 51, 61; stones;

L. populicola (DC.) Duby - 48, 53; Cr, Fr;

L. pulicaris (Pers.) Ach. subsp. pulicaris - 3, 11, 18, 20, 22, 24, 31, 35-40, 42, 43, 47, 53, 54, 65, 68; Al, Aps, Bet, Cav, Cr, Fr, Fs, La, Pa, Ps, Pt, Pav, Q, dw; (Izydorek 2010);

L. rupicola (L.) A. Zahlbr. - 15; stone;

L. saligna (Schrad.) A. Zahlbr. - 52; dw;

L. sarcopidoides (A. Massal.) A.L. Smith - 62; dw;

L. saxicola (Pollich) Ach. - 15, 51, 52, 61, 69; walls, stone; (Izydorek 2010);

L. semipallida H. Magn. - 3, 12, 51; wall;

L. symmicta (Ach.) Ach. - 14, 19, 22, 36, 39, 52, 53; Cr, Ps, Q, dw;

L. thysanophora R.C. Harris - 33; Fs;

L. varia (Hoffm.) Ach. - 17, 29, 39, 40, 52; Pa, dw;

Lecidea fuscoatra (L.) Ach. - 50, 51; stones;

L. nylanderi (Anzi) Th. Fr. - 2, 3, 13, 19, 25, 26, 36-39, 43, 44, 46, 47, 49, 53, 68; Bet, Pa, Ps, Q, U, dw;

Lecidella elaeochroma (Ach.) M. Choisy - 1, 9, 15, 33, 35, 36, 40, 41, 48, 49, 52-56, 61, 64, 65, 68, 69; Ah, Apl, Cav, Cb, Fr, Fs, Pav, Po, Pt, Rr, Q, U; (Izydorek 2010);

L. flavosorediata (Vezda) Hertel \& Leuckert - 11, 28, 29, 48; Aps, Fr, Q;

L. stigmatea (Ach.) Hertel \& Leuckert - 12, 15, 51, 52, 67-69; walls, stone; (Izydorek 2010);

Lepraria eburnea J.R. Laundon - 35; Bet;

L. elobata Tønsberg - 2, 3, 15, 20, 22, 24, 26, 28, 31, 33, 35, 64; Bet, Cb, Fs, Ps, Q, stone;

L. finkii (B. de Lesd.) R.C. Harris - 3, 4, 8-10, 17, 18, 21, 24, 26, 27, 28, 29, 30, 33, 34, 35, 38, 61, 65; Al, Apl, $\mathrm{Cb}, \mathrm{Fr}, \mathrm{Fs}, \mathrm{Pt}, \mathrm{Q}$, Sor;

L. incana (L.) Ach. - 2-4, 7, 8, 16-18, 20, 22, 24-33, 35-39, 42, 46-48, 50, 53, 61, 64, 68; Al, Bet, Cb, Fr, Fs, La, Pa, Ps, Q, Sor, Tc, dw, stone;

L. jackii Tønsberg - 2, 3, 13, 26, 29, 39; Bet, Pa, Ps, Q;

L. neglecta ( Nyl.) Erichsen - 50; stone;

L. rigidula (B. de Lesd.) Tønsberg - 3, 13, 20, 24, 26, 32; Aps, Bet, Fs, Pt, Q; 
L. vouauxii (Hue) R.C. Harris - 8, 15, 35, 45, 51; Al, Apl, Fr, stone;

+Leptorhaphis epidermidis (Ach.) Th. Fr. - 40; Bet;

Lichenomphalia umbellifera (L.) Redhead, Lutzoni, Moncalvo \& Vilgalys - 17, 24, 46; gl, dw;

Melanelixia fuliginosa (Fr. ex Duby) O. Blanco et al. s.str. - 11, 12, 61; stones;

M. glabratula (Lamy) Sandler \& Arup - 1, 3, 4, 7-9, 1728, 30-33, 35, 37-40, 42, 45, 48, 51-55, 62, 64-67; Al, Apl, Aps, Bet, Cav, Cb, Cr, Fr, Fs, Pa, Po, Ps, Pt, Q, Sal, Sor, Tc, dw, stones; (Izydorek 2010);

M. subaurifera (Nyl.) O. Blanco \& al. - 9, 11, 18, 22, 33, 40, 53, 61, 65, 68; Aps, Bet, Cav, Fr, Fs, Ps, Tc, dw;

Melanohalea elegantula (A. Zahlbr.) O. Blanco \& al. - 9, 11, 22, 62; Aps, Fr, Pt, Tc; (Izydorek 2010);

M. exasperatula (Nyl.) O. Blanco \& al. - 6, 7, 9, 11, 15, 40, 42, 48, 49, 51, 52, 61; Apl, Aps, Bet, Fr, La, Q, Tc, $\mathrm{U}, \mathrm{dw}$, metal, stone, eternit, wall;

M. olivacea (L.) Blanco \& al. - 55 (Tobolewski 1981).

Micarea byssacea (Th. Fr.) Czarnota, Guzow-Krzemińska \& Coppins - 35; Cb, Q;

M. denigrata (Fr.) Hedl. - 38, 40, 43, 47, 52, 55; Ps, dw;

M. lithinella (Nyl.) Hedl. - 2, 35; pebbles;

M. micrococca (Körb.) Gams ex Coppins - 25, 26, 31, 37, 38, 39, 44, 46; Al, Bet, Ps, dw;

M. misella (Nyl.) Hedl. - 2, 3, 34, 35, 38, 39, 44, 46, 47; dw;

M. nitschkeana (J. Lahm ex Rabenh.) Harm. - 38, 43, 46; Pa, Ps;

M. prasina Fr. - 2, 33, 39, 40; Aps, Ps, dw;

M. viridileprosa Coppins \& van den Boom - 39, 46; Ps, $\mathrm{dw}$;

Microcalicium disseminatum (Ach.) Vainio - 28, 29; Q;

Ochrolechia androgyna (Hoffm.) Arnold - (Izydorek 2010) (most probably this record belongs to O. bahusienisis);

O. bahusienisis H. Magn. - 37, 39; Q, dw;

O. microstictoides Räsänen - 3, 6, 31, 36-40, 42, 43; Bet, La, Pt, Q, dw;

O. turneri (Sm.) Hasselrot - 15, 45; Aps, Fr;

Opegrapha atra Pers. - 54; Fr;

O. rufescens Pers. - 32; Apl;

O. vulgata (Ach.) Ach. - 30-32; Apl, Q;

Parmelia saxatilis (L.) Ach. s.1. - 8, 9, 32, 36, 37; Bet, Cb, Fr; (Izydorek 2010);

P. serrana A. Crespo, M.C. Molina \& D. Hawksw. - 43; Q;

P. submontana Nádv. ex Hale - 24, Apl;

P. sulcata Taylor - 1, 3, 6, 7, 9, 10, 11 (c.ap.), 13, 15, 18-20, 22, 24, 25, 31-33, 35-42, 45, 46, 48, 49, 51-58, 60, 62, 64-69; Al, Apl, Aps, Bet, Cav, Fr, Fs, La, Pa, Ps, Q, Sal, Tc, U, metal, dw, stones, wall; (Izydorek 2010); Parmelina tiliacea (Hoffm.) Hale - 15, 45, 63; Apl, Fr, Tc; Parmeliopsis ambigua (Wulfen) Nyl. - 2, 3, 19-21,25, 26, 30, 31, 36, 37, 39, 42, 46, 47, 52, 53, 55, 62; Bet, Fs; Pa, Ps, dw; (Izydorek 2010);
Peltigera canina (L.) Willd. - 55; gl;

P. didactyla (With.) J.R. Laundon - 14; gl,

P. hymenina (Ach.) Delise ex Duby - 64; on sand on dune;

P. membranacea (Ach.) Nyl. - 8; stone;

P. polydactylon (Neck.) Hoffm. - 54, 55; gl;

P. praetextata (Flörke) Zopf - 10, 28, stone, dw;

P. rufescens (Weiss) Humb. - 29, 52, 55; dw, gl;

Pertusaria albescens (Huds.) M.Choisy \& Werner - 24, 34, 40, 41, 45, 48, 51, 55, 56, 67; Apl, Aps, Cb, Fr, Fs, Pt; (Izydorek 2010);

P. amara (Ach.) Nyl. - 2, 4, 6-9, 11, 15, 17, 18, 20-22, 25-27, 29-36, 38, 48, 53, 54, 58, 61, 66, 67; Al, Apl, Aps, Cb, Fr, Fs, Q, Sor, Tc; (Izydorek 2010);

P. coccodes (Ach.) Nyl. - 6, 9, 18, 22, 29, 38, 53, 56, 61; Al, Apl, Fr, Fs, Pav, Q; (Izydorek 2010);

P. flavida (DC.) J.R. Laundon - 6, 29, 37, 38, 45; Apl, Fs, Q; (Izydorek 2010);

P. hymenea (Ach.) Schaer. - 4; Fs;

P. leioplaca DC. in Lam. \& DC. - 4, 8, 18, 33-35, 54; Cb, Fr, Fs, Sor; (Izydorek 2010);

P. pertusa (L.) Tuck. - 4, 8, 9, 11, 17, 18, 20, 22, 26, 28, 31, 34, 35, 38, 41, 45; Apl, Aps, Cb, Fr, Fs, Q; (Izydorek 2010);

P. pupillaris (Nyl.) Th. Fr. - 31; Q;

Phaeophyscia nigricans (Flörke) Moberg - 12, 51, 55; walls; (Izydorek 2010);

Ph. orbicularis (Necker) Moberg - 9, 11, 15, 18, 40, 41, 45, 49, 51, 52, 55, 58, 60, 61, 63, 67, 69; Apl, Aps, Fr, Fs, Po, Tc, U, walls, stone; (Izydorek 2010);

Phlyctis argena (Ach.) Flot. - 1, 3, 4, 6-11, 15, 17, 18, 21, 22, 25-28, 32, 34-36, 40, 41, 48, 51-55, 60, 63, 65-67; Al, Apl, Aps, Bet, Cav, Cb, Fr, Fs, Ps, Pt, Q, Sal, Sor, Tc, dw; (Izydorek 2010);

Physcia adscendens (Fr.) H. Olivier - 1, 9, 11, 12, 15, 18, 22, 25, 40, 42, 45, 48, 49, 52, 53, 55, 56, 58, 60, 63-68; Apl, Aps, Bet, Cav, Fr, Fs, La, Po, Ps, Q, Rr, Sal, U, walls; (Izydorek 2010);

Ph. aipolia (Ehrh. ex Humb.) Fürnr. - 9, 32; Apl, Fr;

Ph. caesia (Hoffm.) Fürnr. - 7, 15, 51, 52; metal, stone, wall; (Izydorek 2010);

Ph. dubia (Hoffm.) Lettau - 11, 51, 52, 66, 67; Apl, Aps, walls;

Ph. stellaris (L.) Nyl. - 9, 36, 48, 49, 65; Fr, Q, U;

Ph. tenella (Scop.) DC. - 1, 6, 8, 9, 11, 18, 25, 26, 35, 36, 37, 40, 41, 42, 45, 48, 49, 52, 53, 54, 55, 56, 60, 63-68; Apl, Aps, Bet, Cav, Cr, Fr, Fs, Pa, Po, Ps, Q, Sal, Tc, U, dw, wall; (Izydorek 2010);

Physconia distorta (With.) J.R. Laundon - 1, 11, 40, 45, 56, 61; Apl, Aps, Fr, Po, Pt; (Izydorek 2010);

Ph. enteroxantha (Nyl.) Poelt - 9, 11, 25, 48; Apl, Aps, Fr; (Izydorek 2010);

Ph. grisea (Lam.) Poelt - 11, 48, 60, 61; Apl, Aps, Fr;

Ph. perisidiosa (Erichsen) Moberg - 11, 61; Apl, Aps; 
Placynthiella dasaea (Stirt.) Tønsberg - 2, 3, 14, 15, 19, 24-26, 30, 31, 36, 40, 46, 62, 68; Bet, Fs, Q, dw, humus;

P. icmalea (Ach.) Coppins \& P. James - 3, 20, 21, 27, 37, 46, 62; Bet, dw;

P. oligotropha (J.R. Laundon) Coppins \& P. James - 37, 46; gl, humus; (Izydorek 2010);

P. uliginosa (Schrader) Coppins \& P. James - 37, 43, 54, 55; Bet, gl, dw; (Izydorek 2010);

Platismatia glauca (L.) W.L. Culb. \& C.F. Culb. - 2, 3, 6, $10,13,20,21,24-26,29,31,33,35,36,38-40,42$, 46, 52, 54, 55, 62; Al, Bet, Cav, Fs, La, Pa, Ps, Q, Sor, dw; (Izydorek 2010);

Pleurosticta acetabulum (Necker.) Elix \& Lumbsch - 11, 15, 40-42, 45, 48, 55, 56, 60, 61; Apl, Aps, Fr, Po, Q, Tc; (Izydorek 2010);

Polycauliona candelaria (L.) Frödén, Arup \& Søchting - 11, 22, 26, 45, 58, 66; Apl, Aps, Fs, La; (Izydorek 2010);

P. polycarpa (Hoffm.) Frödén, Arup \& Søchting - 1, 17, 19, 35, 37, 41, 42, 45, 48, 49, 52, 54, 60, 63, 65; Apl, Aps, Bet, Cav, Fr, Pa, Pt, Q, Sal, Tc, U, wall, dw, polypore;

Polysporina simplex (Davies) Vězda - 12, 51; stones;

Porina aenea (Wallr.) A. Zahlbr. - 4, 31, 32, 33, 54, 67; Aps, Cb, Fr, Fs, Pav;

P. chlorotica (Ach.) Hafellner \& Kalb - 10, 35; stones;

Porpidia crustulata (Ach.) Hertel \& Knoph - 26, 51, 52; wall, stones;

P. macrocarpa (DC.) Hertel \& A.J. Schwab - 31; stone;

P. soredizodes (Lamy ex Nyl.) J. R. Laundon - 10, 15; stone;

P. tuberculosa (Sm.) Hertel \& Knoph - 10, 51, 52; stones, wall;

Pseudevernia furfuracea (L.) Zopf - 2, 3, 6, 7, 13, 14, 16, 19-21, 29, 31, 36, 37, 39, 40, 42, 44, 46, 52, 62, 66; Apl, Bet, Fs, La, Pa, Pm, Ps, metal, dw; (Izydorek 2010);

Psilolechia lucida (Ach.) M. Choisy - 51, 63, 67; stone, walls;

Punctelia jeckeri (Roum.) Kalb - 15, 51, 60; Apl, Fr;

P. subrudecta (Nyl.) Krog - 15; Apl, Fr;

Pycnora sorophora (Vain.) Hafellner - 40, 43, 44, 47; Ps, $\mathrm{dw}$;

Pyrenula nitida (Weigel) Ach. - 8; Cb;

Pyrrhospora quernea (Dicks.) Körb. - 18; Fr;

Ramalina baltica Lettau - 61, Apl;

R. farinacea (L.) Ach. - 2, 3, 6, 7, 9, 11 (c.ap.), 15, 18, 22, 29, 36, 38, 41, 42, 45, 49, 51-53, 55, 56, 58, 60, 61, 64-67; Apl, Aps, Cav, Fr, Fs, Pm, Ps, Pt, Q, Tc, U, dw, stone; (Izydorek 2010);

R. fastigiata (Pers.) Ach. - 11, 15,38, 41, 45, 48, 51, 55, 56, 58, 59, 60, 61, 65-67; Apl, Aps, Cav, Fr, Q; (Izydorek 2010);
R. fraxinea (L.) Ach. - 11, 40, 41, 45, 48, 51, 56, 58, 59, 61, 65-67; Apl, Aps, Fr, Po, Q; (Izydorek 2010);

Reichlingia leopoldii Diederich \& Scheid. - 20; Q;

Rhizocarpon geographicum (L.) DC. - 51; głaz, wall;

Rinodina oleae Bagl. - 15, 67; walls;

R. pyrina (Ach.) Arnold - 45; Aps;

Ropalospora viridis (Tønsberg) Tønsberg - 3, 4, 17, 2022, 26-28, 30-35, 38; Aps, Bet, Cav, Cb, Fs, Q;

Rusavskia elegans (Link) S.Y. Kondr. \& Karnefelt - 24, 52; Apl, concrete;

Scoliciosporum chlorococcum (Graewe ex Stenh.) Vězda - 22, 31, 42, 43, 46, 52, 53, 62; Bet, Pa, Pm, Ps, dw; (Izydorek 2010);

S. sarothamnii (Vain.) Vězda - 37; Pa;

S. umbrinum (Ach.) Arnold - 15, 23, 37, 42, 51, 52; stones, wall;

Strangospora pinicola (A. Massal.) Koerb. - 39, 52; dw;

Tephromela atra (Huds.) Hafellner - 15; stone;

Trapelia coarctata (Sm.) Choisy - 25, 29, 31, 55, 67; stone, walls;

T. obtegens (Th. Fr.) Hertel - 15; stone;

T. placodioides Coppins \& P. James - 10, 52; stones;

Trapeliopsis aeneofusca (Flörke) Coppins \& P. James (acc. Izydorek 2010);

T. flexuosa (Fr.) Coppins \& P. James - 2, 3, 14, 21, 26, 31, 36, 40, 42-44, 46, 52; Bet, Ps; dw; (Izydorek 2010);

T. granulosa (Hoffm.) Lumbsch - 2, 3, 13, 14, 19, 20, 22, 31, 37, 39, 40, 43, 46, 55, 62, 68; Bet, Ps, Q, dw; (Izydorek 2010);

T. pseudogranulosa Coppins \& P. James - 20, 24, 26, 29, 31, 34, 35; Bet, gl, dw;

Usnea dasopoga (Ach.) Röhl. - 3, 6, 32, 36, 38, 52; Bet, Fs, La, Pa, Q; (Izydorek 2010);

U. florida (L.) Weber ex F.H. Wigg. - 3, 6, 9, 13, 32, 37, 39, 40, 43, 44, 47, 52, 62; Bet, Fr, La, Pa, Ps, Q, dw; (Izydorek 2010);

U. hirta (L.) Weber ex F.H. Wigg. - 19, 37, 39, 40, 43, 44, 47, 52, 62; Bet, La, Ps, Q, dw; (Izydorek 2010);

Varicellaria hemisphaerica (Flörke) Schmitt \& Lumbsch 9, 17, 26-29, 38; Al, Fs, Q; (Izydorek 2010);

Verrucaria nigrescens Pers. - 47, 51, 52; wall, stone; (Izydorek 2010);

V. praetermissa (Trevis.) Anzi - 8; stone;

Violella fucata (Stirt.) T. Sprib. - 2, 3, 8, 13, 16, 18-20, 24-27, 30, 31, 33-36, 39, 40, 42-44, 47; Al, Bet, Cav, $\mathrm{Cb}, \mathrm{Pa}, \mathrm{Fs}, \mathrm{Ps}, \mathrm{Q}, \mathrm{dw}$, twigs of Ledum palustre;

Vulpicida pinastri (Scop.) J.E. Mattsson \& Lai - 6, 22, 46; La, Pa; (Izydorek 2010);

Xanthoparmelia conspersa (Ach.) Hale - 12, 26, 48, 51; stones;

$X$. delisei (Duby) Essl. - 50, 51; stones;

$X$. loxodes (Nyl.) O. Blanco et al. - 15, 27; stones;

Xanthoria parietina (L.) Th. Fr. - 1, 7, 9, 11, 15, 18, 19, 36, 40, 42, 45, 48, 49, 51, 52, 54-56, 60-69; Apl, Aps, 
Bet, Fr, Po, Ps, Pt, Ra, Q, Sal, Tc, U, dw, metal, wall, eternit; (Izydorek 2010);

Zwackhia viridis (Ach.) Poetsch \& Schied. - 8, 18, 20, 22; Cb, Fr, Fs, Q, Tc; (Izydorek 2010).

\section{Conclusions}

The Karnieszewice Forest Division, despite forest management carried out since many years, is relatively in a good condition and characterized by high average of the tree stand age. Additionaly, in this area a strong air pollution were never detected. Simultanously, habitat conditions (among others high air and substrates humidity) are very convenient for the lichen vegetation, especially for epiphytic species. Presented results can prove that in this case impact of forest management on lichen biota is not as drastic as it has been described in the literature. However, there are not many lichens typical for primary forests or they are rare.

In our opinion, rational forest culture based on ecological rules and preservation of forest continuity on larger areas enable the occurrence of numerous stenotopic lichen species, sensitive to changes of habitat conditions.

\section{Acknowledgements}

The authors would like to express their gratitude to workers of the Karnieszewice Forest Inspectorate, especially Dagny Nowak-Staszewska, Jarosław Staniszewski and Tomasz Kapustyński, for their help during the fieldwork and financial support of this work. We are thankful to Reviewers for their favorable review.

\section{References}

Adamska E., 2014, Biota porostów Torunia na tle warunków siedliskowych miasta. [Lichen biota in Toruń in relation to habitat conditions of the city], Wyd. Naukowe UMK, Toruń.

Arcadia L., 2013, Usnea dasopoga, a name to be reinstated for U. filipendula, and its orthography, Taxon 62(3): 604-605.

Arcadia L. \& Knudsen K., 2012, The name Myriospora is available for the Acarospora smaragdula group, Opuscula Philolichenum 11: 19-25.

Arup U. \& Sandler Berlin E., 2011, A taxonomic study of Melanelixia fuliginosa in Europe, Lichenologist 43(2): 89-97.

Arup U., Søchting U. \& Frödén P., 2013, A new taxonomy of the family Teloschistaceae, Nord. J. Bot. 31: 16-83.
Bezmian, A., 1998, Porosty Koszalina na tle warunków klimatyczno-antropogenicznych [Lichens of Koszalin in climatic and anthropogenic terms], [in:] J. Miądlikowska (ed.) Botanika polska u progu XXI wieku, Materiały sympozjum i obrad sekcji 51 Zjazdu Polskiego Towarzystwa Botanicznego, Gdańsk, 15-19 września 1998, [Polish botany on the threshold of the XXI century, Proceedings of the 51 Congress of the Polish Botanical Society, Gdańsk, 15-19 September 1998], Wyd. Nauk. Boguccy, Poznań: 36.

Brodo I. M., Owe-Larsson B. \& Lumbsch H. T., 1994, The sorediate, saxicolous species of the Lecanora subfusca group in Europe, Nord. J. Bot. 14(4): 451-461.

Cieśliński S., 2003, Atlas rozmieszczenia porostów (Lichenes) w Polsce północno-wschodniej [Distribution atlas of lichens (Lichenes) in north-eastern Poland], Phytocoenosis 15(N.S.), Suppl. Cartographiae Geobotanicae 15: $1-430$.

Cieśliński S., Czyżewska K. \& Fabiszewski J., 2006, Red list of the lichens in Poland, [in:] Z. Mirek, K. Zarzycki, W. Wojewoda, Z. Szeląg (eds) Red list of plants and fungi in Poland, W. Szafer Institute of Botany, Polish Academy of Sciences, Kraków: 71-89.

Czyżewska K., 1976, Zanikanie porostów epifitycznych pod wpływem antropogenicznej deegeneracji lasów liściastych Puszczy Pilickiej [Dissapearance of epiphytic lichens under the influence of antropogenic degenerations of broadleaved forests in the Pilicka Forest complex], Phytocoenosis 5(3-4): 363-375.

Ekman S. \& Svensson M., 2014, Brianaria, a new genus to accommodate the Micarea sylvicola group, The Lichenologist 46: 285-294.

Erichsen C.F.E., 1936, Pertusariaceae, [in:] Rabenhorst's Kryptogamen-Flora von Deutschland, Õsterreich und der Schweiz 9.5(1): 321-728, Leipzig.

Erichsen C. F. E., 1940, Lichenologische Beiträge. III, Ann. Mycol. 38.2-4: 303-331.

Ertz D. \& Tehler A., 2011, The phylogeny of Arthoniales (Pezizomycotina) inferred from nucLSU and RPB2 sequences, Fung. Diversity 49(1): 47-71.

Fałtynowicz W., 1992, The lichens of Western Pomerania (NW Poland), An ecogeographical study, Polish Bot. Stud. 4: 1-182.

Fałtynowicz W., 1995, Wykorzystanie porostów do oceny zanieczyszczenia powietrza [Use of lichens for estimating of air pollution], Fundacja CEEW, Krosno.

Fałtynowicz W. \& Kukwa M., 2006, Lista porostów i grzybów naporostowych Pomorza Gdańskiego [List of lichens and lichenicolous fungi of Gdańsk Pomerania], Acta Bot. Cassubica, Monographiae 2: 1-98.

Hawksworth D. L., 1971, Lichens as litmus for air pollution: a historical review, Intern. J. Environmental Studies 1: 281-296. 
Herzig R. \& Urech M., 1991, Flechten als Bioindicatoren. Integriertes biologisches Messystem der luftverschmutzung für Schweizer Mitteland, Biblioth. Lichenol. 43: $1-283$.

Izydorek I., 2010, Porosty Wysoczyzny Polanowskiej [The lichens of Wysoczyzna Polanowska district], Słupskie Prace Biol. 7: 51-78.

Jóźwiak M.A., 2014, Wykorzystanie organizmów wskaźnikowych $\mathrm{w}$ bioindykacji środowisk lądowych i wodnych na wybranych przykładach [The use of indicative organisms in bioindication of land and water environments with the chosen examples], Kieleckie Tow. Nauk., Kielce.

Kalb K., 2007, New or otherwise interesting lichens, Biblioth. Lichenol. 95: 297-316.

Krawiec F., 1934, Flora epifityczna lasów bukowych Wielkopolski [Die Epithyten Flora der Buchenwälder Grosspolens], Acta Soc. Bot. Pol. 11 (Suppl.): 317-327.

Kubiak D., Czarnota P., Zduńczyk A., Dynowska M., Leśniański G., Grabowska A., Olszewska S., Sadowska-Deś A. \& Wojdal P., 2014, The preservation status of the lichen biota in the designed Special Area of Conservation NATURA 2000 ,Middle Łyna River Valley - Smolajny" (the Forest Division of Wichrowo), Acta Mycol. 49(1): 135-146.

Kukwa M., Kowalewska A., Śliwa L., Czarnota P., Czyżewska K., Flakus A., Kubiak D., Wilk K., Dimos-Zych M., Kolanko K., Szymczyk R., Lipnicki L., Adamska E., Bielec D., Guzow-Krzemińska B., Gruszka W., Hachułka M., Jabłońska A., Oset M., Kiszka J., Kozik J., Leśniański G. \& Lazarus M., 2012, Porosty i grzyby naporostowe Wdzydzkiego Parku Krajobrazowego (Pomorze Gdańskie, N Polska) [Lichens and lichenicolous fungi of the Wdzydzki Landscape Park (Pomorze Gdańskie, N Poland)], Acta Bot. Cassubica 11: 75-103.

Kukwa M., Fałtynowicz W., Kowalewska A., Szymczyk R., Adamska E. \& Pietrzykowska K., 2013, Materiały do bioty porostów i grzybów naporostowych nadleśnictwa Karnieszewice i okolic (Pomorze Zachodnie), Piąte Dni Różnorodności Biologicznej w Leśnym Kompleksie Promocyjnym Lasy Środkowopomorskie [Materials to the lichen biota and lichenicolous fungi of the Karnieszewice Forest Division (Western Pomerania)], Wyd. EKWITA, Gdańsk: 9-17.

Laundon J. R., 2010, Lecanora antiqua, a new saxicolous species from Great Britain, and the nomenclature and authorship of L. albescens, L. conferta and L. wallalis, Lichenologist 42(6): 631-636.

Lendemer J. C., 2011, A standardized morphological terminology and descriptive scheme for Lepraria (Stereocaulaceae), Lichenologist 43(5): 379-399.

Łubek A., 2007, Antropogeniczne przemiany bioty porostów Świętokrzyskiego Parku Narodowego i otu- liny [Anthropogenic changes of lichen biota of the Świętokrzyski National Park and its protective zone], Fragm. Flor. Geobot., Ser. Polonica, Supplementum 10: 3-94.

Motyka J., 1934, W sprawie ochrony porostów [On the protection of lichens], Ochr. Przyr. 14: 50-56.

Neuwirth G. \& Aptroot A., 2011, Recognition of four morphologically distinct species in the Graphis scripta complex in Europe, Herzogia 24(2): 207-230.

Nordin A., Savić S. \& Tibell L., 2010, Phylogeny and taxonomy of Aspicilia and Megasporaceae, Mycologia 102(6): 1339-1349.

Nylander W., 1866, Les lichens du Jardin du Luxembourg, Bull. Soc. Bot. Fr. 13: 364-372.

Orange A., James P. W. \& White F. J., 2001, Microchemical methods for the identification of lichens, British Lichen Society, London.

Palice Z., Printzen C., Spribille T. \& Elix J. A., 2011, Notes on the synonyms of Lecanora filamentosa, Graphis Scripta 23(1): 1-7.

Rozporządzenie Ministra Środowiska z dnia 16 października 2014 r. w sprawie ochrony gatunkowej grzybów, Dz. U., poz. 1408 [Regulation of the Minister of the Environment of 16 October 2014 on the protection of fungi species, Journal of Laws no. 1408], 2014.

Santesson R., Moberg R., Nordin A., Tønberg T. \& Vitikainen O., 2004, Lichen-forming and lichenicolous fungi of Fennoscandia, Museum of Evolution, Uppsala University, Uppsala.

Schiefelbein U., Czarnota P., Thüs H. \& Kukwa M., 2012, The lichen biota of the Drawieński National Park (NW Poland, Western Pomerania), Folia Cryptog. Estonica 49: 59-71.

Schmitt I., Otte J., Parnmen S., Sadowska-Deś A.D., Lücking R. \& Lumbsch H. T., 2012, A new circumscription of the genus Varicellaria (Pertusariales, Ascomycota), MycoKeys 4: 23-36.

Spribille T., Goffinet B., Klug B., Muggia L., Obermayer W. \& Mayrhofer H., 2011, Molecular support for the recognition of the Mycoblastus fucatus group as the new genus Violella (Tephromelataceae, Lecanorales), Lichenologist 43(5): 445-466.

Tobolewski Z., 1964, Lichenotheca Polonica, Fasc. XVII, No. 376-400, Lichenes Poloniae Septentrionali-occidentalis, Wyd. PAN, Poznań.

Tobolewski Z., 1966, Rodzina Caliciaceae (Lichenes) w Polsce [The family Caliciaceae (Lichenes) in Poland], Prace Komis. Biol. Pozn. Tow. Przyj. Nauk 24(5): 1-101.

Tobolewski Z., 1979, Porosty (Lichenes) 5, Atlas rozmieszczenia roślin zarodnikowych w Polsce, ser. III [Atlas of geographical distribution of spore-plants in Poland, ser. III.], PWN, Warszawa-Poznań. 
Tobolewski Z., 1980, Porosty (Lichenes) 6, Atlas rozmieszczenia roślin zarodnikowych w Polsce, ser. III [ Atlas of geographical distribution of spore-plants in Poland, ser. III], PWN, Warszawa-Poznań.

Tobolewski Z., 1981, Porosty (Lichenes) 7, Atlas rozmieszczenia roślin zarodnikowych w Polsce, ser. III [Atlas of geographical distribution of spore-plants in Poland, ser. III], PWN, Warszawa-Poznań.

van Herk C. M., 2001, Bark pH and susceptibility to toxic air pollutants as independent causes of changes in epiphytic lichen composition in space and time, Lichenologist 33(5): 419-441.

Wieczorek A., 2005, Biota porostów Szczecińskiego Parku Krajobrazowego [Lichen flora of the Szczecin Landscape Park], Fragm. Flor. Geobot. Polonica 12(1): $143-156$.

Wieczorek A., 2014, Operat ochrony porostów Wolińskiego Parku Narodowego [Statement of lichens protection in the Woliński National Park], Mscr., Szczecin.
Zalewska A., 2012, Ecology of lichens of the Puszcza Borecka Forest (N Poland), W. Szafer Institute of Botany, Polish Academy of Sciences, Kraków.

Zalewska A., Fałtynowicz W., Krzysztofiak A., Krzysztofiak L. \& Picińska-Fałtynowicz J., 2004a, Lichens of the Suwalski Landscape Park, [in:] A. Zalewska, W. Fałtynowicz (eds) Lichens of the protected areas in the Euroregion Niemen, Stowarzyszenie „Człowiek i Przyroda", Suwałki: 5-50.

Zalewska A., Fałtynowicz W., Krzysztofiak A., Krzysztofiak L. \& Picińska-Fałtynowicz J., 2004b, Lichens of Romincka Primeval Forest, [in:] A. Zalewska, W. Fałtynowicz (eds) Lichens of the protected areas in the Euroregion Niemen, Stowarzyszenie „Człowiek i Przyroda", Suwałki: 51-109. 